\title{
On the Origin of Hybrid Imaging
}

\author{
Adriaan A. Lammertsma \\ Department of Radiology and Nuclear Medicine, Amsterdam University Medical Centers, location VUmc, Amsterdam, The Netherlands
}

$\mathbf{P}$ There is no question that ${ }^{18} \mathrm{~F}$-FDG PET imaging is the most sensitive method for staging of cancers. In addition, PET perfusion imaging is the most accurate method for selecting patients who will benefit from a heart catheterization procedure. Finally, amyloid imaging is taking hold as a valuable tool in the differential diagnosis of Alzheimer disease, and new applications, such as the evaluation of inflammatory processes in rheumatoid arthritis, are constantly emerging. The evolution of PET from a prototype physiologic measurement tool to its widespread use in diagnostic medicine is the result of several technologic innovations in combination with a constantly increasing armamentarium of PET tracers, enabling the assessment of an ever-increasing number of molecular processes.

Received Jul. 1, 2020; revision accepted Aug. 27, 2020

For correspondence or reprints contact: Adriaan A. Lammertsma, Department of Radiology and Nuclear Medicine, Amsterdam University Medical Centers, location VUmc, P.O. Box 7057, 1007 MB Amsterdam, The Netherlands.

E-mail: aa.lammertsma@amsterdamumc.nl

COPYRIGHT @ 2020 by the Society of Nuclear Medicine and Molecular Imaging. DOI: 10.2967/jnumed.120.252841
TABLE 1

Key Innovations in Development of PET

\begin{tabular}{cl}
\hline Year & \multicolumn{1}{c}{ Key innovation } \\
\hline 1975 & First working prototype scanner \\
1978 & First commercial scanner \\
1989 & First 3-dimensional studies \\
1992 & First description of whole-body scans \\
2000 & First PET/CT scanner \\
2017 & First total-body PET scanner \\
\hline
\end{tabular}

Although tracer development is important in the search for more selective diagnostic procedures, there is little doubt that the field would not have survived without ${ }^{18} \mathrm{~F}-\mathrm{FDG}$. Having acknowledged the importance of radiochemical developments, this contribution will focus on technologic developments, with the key innovations summarized in Table 1.

Clearly, none of the developments listed in Table 1 would have been possible without the initial construction of a working PET scanner. Although there had been earlier attempts, which in general

\section{A Combined PET/CT Scanner for Clinical Oncology}

Thomas Beyer, David W. Townsend, Tony Brun, Paul E. Kinahan, Martin Charron, Raymond Roddy, Jeff Jerin, John Young, Larry Byars, and Ronald Nutt

PET Facility and Division of Nuclear Medicine, Department of Radiology, University of Pittsburgh, Pittsburgh, Pennsylvania; CTI PET Systems, Knoxville; and Byars Consulting, Oak Ridge, Tennessee

The availability of accurately aligned, whole-body anatomical (CT) and functional (PET) images could have a significant impact on diagnosing and staging malignant disease and on identifying and localizing motastasos. Computor algonthms to align CT and ful for the whe wheres ima alignment in other regions of the body is mor problem graph with the unique capability of acquiring accurately alignofunctional and anatomical images for any part of the humanged has been designed and built. The PET/CT scanner was devel oped as a combination of a Siemens Somatom AR.SP spiral CT and a partial-ring, rotating ECAT ART PET scanner. All components are mounted on a common rotational support within single gantry. The PET and CT components can be operated either separately, or in combined mode. In combined mode, the CT images are used to correct the PET data for scatter and attenuation. Fully quantitative whole-body images are obtained for an axial extent of $100 \mathrm{~cm}$ in an imaging time of less than $1 \mathrm{~h}$. When operated in PET mode alone, transmission scans are acquired with dual ${ }^{3 r} \mathrm{Cs}$ sources. Results: The scanner is fully operational and the combined device has been operated success. fully in a clinical environment. Over 110 patients have been imaged, covering a range of different cancers, including lung. esophageal, head and neck, melanoma, lymphoma, pancreas, and renal cell. The aligned PET and CT images are used both for diagnosing and staging disease and for evaluating response to therapy. We report the first pertormance measurements from the scanner and prosent some illustrative clinical studies acquiredin a protical and effective approm to ace anatomical-based modalities, such as $\mathrm{x}$-ray $\mathrm{CT}$ and MR. Malignant cells have increased facilitated glucose transpor and upregulation of hexokinase activity, and hence tumors can be identified by regions of increased glucose utilization (1). The PET tracer FDG of increased glucose utilization lucose metabolism, a glucose analog, is used to image increased FDG uptake are considered suspicious for malignant disease, particularly as metabolic changes often precede the morphological changes associated with disease (2). Disense managent will depend on the with disease (2) Disease mager the metastases identified at the time of presentation. Therefore, whole-body FDG PET scanning, which can be used to diagnose and stage primary malignancy and to localize disseminated, metastatic disease in almost any region of the body, is becoming a standard procedure for imaging cancer (3).

One area in which FDG PET can play a significant role is in establishing response to treatment $(l)$. Current procedures to monitor therapy use mainly anatomical imaging modaliies, such as $\mathrm{CT}$, even though metabolic changes in tumos ches. A significant metabolic change can be este by comparing uptake values from pre- and posttreatmen scans, although such comparisons can only be made accurately on attenuation-corrected, quantitative PET images. Attenuation correction for whole-body scanning has gener-

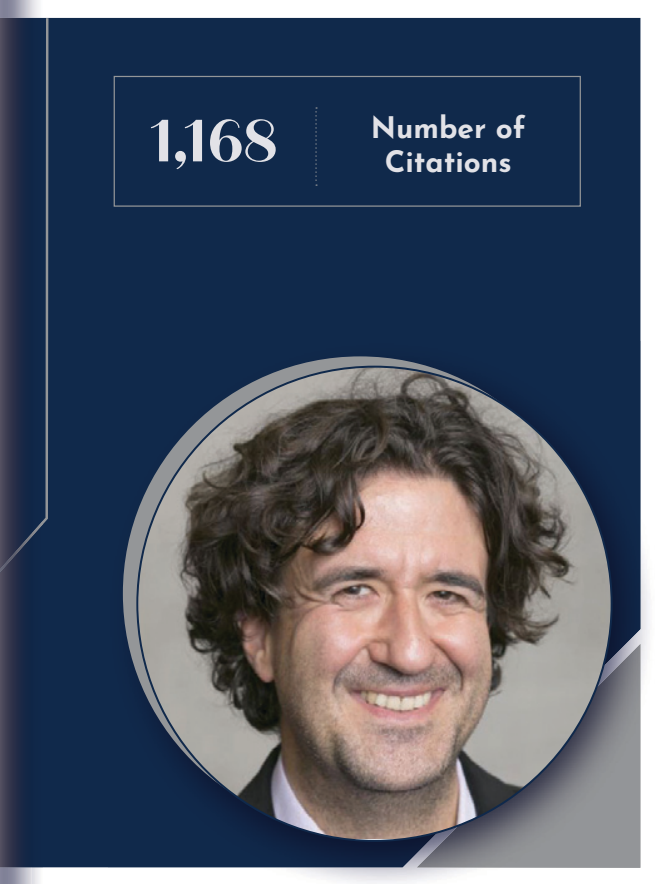


were not successful because of a too-limited number of projections, the first working PET scanner was constructed by the St. Louis group $(1,2)$. Subsequently, 2 key scientists of this team (Phelps and Hoffman) moved to Los Angeles, where a PET scanner was built

(3) that was subsequently commercialized.

The first generation of commercial PET scanners consisted of relatively large sodium iodide crystals. To achieve a sufficient number of projections, detectors underwent rotational and translational movements. For a complete scan, the mechanical movements required approximately $30 \mathrm{~s}$, precluding acquisition of short scans at the beginning of a dynamic scan. Next, the emergence of new crystal materials made it possible to construct scanners with stationary rings and with sufficient sensitivity to enable acquisition of dynamic scans. To limit scatter in subsequent multiring devices, tungsten septa were positioned between rings. When methods to correct for scatter improved, it was possible to remove these septa, allowing for so-called 3-dimensional scans (4), which meant a major increase in sensitivity.

The developments mentioned above all happened in a period of approximately 20 years when PET was embedded primarily in the research domain (apart from its use in assessing myocardial viability). In the mid-1990s, however, a new development-the possibility of surveying the whole body (whole-body scan) (5) rekindled interest in oncology, and many subsequent studies showed that ${ }^{18} \mathrm{~F}$-FDG PET was the most sensitive method to detect metastases, with obvious (and now well-known) implications for staging and preventing futile surgery.

Whole-body ${ }^{18} \mathrm{~F}$-FDG PET for tumor staging became the key factor in establishing PET as a clinical diagnostic tool, and at the end of the 1990s there was a sharp increase in PET sales. Despite the progress in staging, it was not always easy to determine the exact sites of lesions, as the transmission scan did not provide enough anatomic information and coregistration with a separately acquired CT scan proved to be difficult, at least in some parts of the body. In addition, although the transmission scan may be the purest and most accurate method for measuring tissue attenuation, it suffers from poor statistics. Consequently, the duration of the transmission scan became a burden both for the patient and for patient throughput.

The solution for this problem was as brilliant as it was simple. David Townsend (then at Geneva) and Ron Nutt (from CTI, the main PET manufacturer at the time) had developed a cheaper type of PET scanner, one with a partial ring of detectors (which were and still are the most expensive part of a scanner) rotating around the patient. Given the continuous call for more anatomic information for staging purposes and the realization that there was a lot of empty space in this rotating PET camera, they had the idea to use the opposite part of the ring for mounting a CT scanner.

The idea to mount both PET and CT on the same ring proved to be less practical, but they pursued their idea by putting 2 separate rings together, resulting in the first combined PET/CT scanner. An article about this scanner was published in The Journal of Nuclear Medicine and became the highest-cited paper in that decade (6).

Interestingly, the rotating PET camera, developed to save costs, was not a commercial success, but the combination of PET and CT in a single scanner was. No longer were PET scanners sold primarily to research groups; instead, the combination of PET and CT scanners in a single machine became a driving force in making PET attractive for clinical sites. All subsequent full-ring PET scanners were equipped with a CT component, and within 10 years after the description of the first PET/CT scanner, it was no longer possible to buy a PET-alone scanner.

The next major innovation in PET was the so-called total-body PET scanner (7). It is needless to say that these scanners will also be equipped with CT, illustrating that PET/CT is not a short-lived hype but an idea-a development that will stay with us for the foreseeable future.

\section{DISCLOSURE}

No potential conflict of interest relevant to this article was reported.

\section{REFERENCES}

1. Phelps ME, Hoffman EJ, Mullani NA, Ter-Pogossian MM. Application of annihilation coincidence detection to transaxial reconstruction tomography. $\mathrm{J} \mathrm{Nucl}$ Med. 1975;16:210-224.

2. Ter-Pogossian MM, Phelps ME, Hoffman EJ, Mullani NA. A positron emission transaxial tomograph for nuclear medicine imaging (PETT). Radiology. 1975;114: 89-98.

3. Phelps ME, Hoffman EJ, Huang SC, Kuhl DE. ECAT: a new computerized tomographic imaging system for positron-emitting radiopharmaceuticals. $\mathrm{J} \mathrm{Nucl}$ Med. 1978;19:635-647.

4. Townsend DW, Sprinks T, Jones T, et al. Three dimensional reconstruction of PET data from a multi-ring camera. IEEE Trans Nucl Sci. 1989;36:1056-1065.

5. Dahlbom M, Hoffman EJ, Hoh CK, et al. Whole-body positron emission tomography: part I. methods and performance characteristics. J Nucl Med. 1992;33: 1191-1199.

6. Beyer T, Townsend DW, Brun T, et al. A combined PET/CT scanner for clinical oncology. J Nucl Med. 2000;41:1369-1379.

7. Cherry SR, Jones T, Karp JS, Qi J, Moses WW, Badawi RD. Total-body PET: maximizing sensitivity to create new opportunities for clinical research and patient care. J Nucl Med. 2018;59:3-12. 


\title{
A Combined PET/CT Scanner for Clinical Oncology
}

\author{
Thomas Beyer, David W. Townsend, Tony Brun, Paul E. Kinahan, Martin Charron, Raymond Roddy, Jeff Jerin, \\ John Young, Larry Byars, and Ronald Nutt \\ PET Facility and Division of Nuclear Medicine, Department of Radiology, University of Pittsburgh, Pittsburgh, Pennsylvania; CTI \\ PET Systems, Knoxville; and Byars Consulting, Oak Ridge, Tennessee
}

\begin{abstract}
The availability of accurately aligned, whole-body anatomical (CT) and functional (PET) images could have a significant impact on diagnosing and staging malignant disease and on identifying and localizing metastases. Computer algorithms to align CT and PET images acquired on different scanners are generally successful for the brain, whereas image alignment in other regions of the body is more problematic. Methods: A combined PET/CT tomograph with the unique capability of acquiring accurately aligned functional and anatomical images for any part of the human body has been designed and built. The PET/CT scanner was developed as a combination of a Siemens Somatom AR.SP spiral CT and a partial-ring, rotating ECAT ART PET scanner. All components are mounted on a common rotational support within a single gantry. The PET and CT components can be operated either separately, or in combined mode. In combined mode, the CT images are used to correct the PET data for scatter and attenuation. Fully quantitative whole-body images are obtained for an axial extent of $100 \mathrm{~cm}$ in an imaging time of less than $1 \mathrm{~h}$. When operated in PET mode alone, transmission scans are acquired with dual ${ }^{137} \mathrm{Cs}$ sources. Results: The scanner is fully operational and the combined device has been operated successfully in a clinical environment. Over 110 patients have been imaged, covering a range of different cancers, including lung, esophageal, head and neck, melanoma, lymphoma, pancreas, and renal cell. The aligned PET and CT images are used both for diagnosing and staging disease and for evaluating response to therapy. We report the first performance measurements from the scanner and present some illustrative clinical studies acquired in cancer patients. Conclusion: A combined PET and CT scanner is a practical and effective approach to acquiring co-registered anatomical and functional images in a single scanning session.
\end{abstract}

Key Words: oncology; PET; CT; dual modality; image fusion

J Nucl Med 2000; 41:1369-1379

DOI: 10.2967/jnumed.106.252841a

$\mathbf{T}$ he role of PET imaging in clinical oncology and patient care is increasing. Clinical decisions based on PET studies are changing cancer patient management by adding unique functional information to that obtained from conventional anatomical-based modalities, such as x-ray CT and MR. Malignant cells have increased facilitated glucose transport and upregulation of hexokinase activity, and hence tumors can be identified by regions of increased glucose utilization (1). The PET tracer FDG, a glucose

Received Jul. 7, 1999; revision accepted Sep. 21, 1999.

For correspondence or reprints contact: David W. Townsend, PhD, PET Facility, Department of Radiology, University of Pittsburgh, 200 Lothrop St., Pittsburgh, PA 15213.

COPYRIGHT (C 2020 by the Society of Nuclear Medicine and Molecular Imaging. analog, is used to image glucose metabolism in patients. Focal areas of abnormally increased FDG uptake are considered suspicious for malignant disease, particularly as metabolic changes often precede the morphological changes associated with disease (2). Disease management will depend on the tumor type, the extent and aggressiveness of the lesion, and on local and distant metastases identified at the time of presentation. Therefore, wholebody FDG PET scanning, which can be used to diagnose and stage primary malignancy and to localize disseminated, metastatic disease in almost any region of the body, is becoming a standard procedure for imaging cancer (3).

One area in which FDG PET can play a significant role is in establishing response to treatment (1). Current procedures to monitor therapy use mainly anatomical imaging modalities, such as CT, even though metabolic changes in tumors may occur earlier than, or even instead of, anatomical size changes. A significant metabolic change can be established by comparing uptake values from pre- and posttreatment scans, although such comparisons can only be made accurately on attenuation-corrected, quantitative PET images. Attenuation correction for whole-body scanning has generally been problematic because of the increase in image noise from the transmission scan and the increase in total scan duration (emission plus transmission), which may be difficult for certain patients to tolerate for up to $1 \mathrm{~h}$ at a time. Therefore, many clinical readings are still performed on images uncorrected for attenuation.

A difficulty for the interpretation of FDG PET scans, particularly in the abdomen, is the absence of identifiable anatomical structures. The low contrast and low resolution anatomy visualized in the PET scan is insufficient for precise anatomical localization of foci of abnormal uptake. However, localization of increased FDG uptake to a specific organ or structure can be important when decisions affecting the diagnosis, staging, and treatment of the patient are to be made. The significance of additional anatomical information from CT has been recognized in oncology (4), but computer algorithms to co-register functional and anatomical images, although successful for relatively fixed organs such as the brain (5), are less satisfactory for internal abdominal organs that can move independently between scans. In such situations, no linear transformation exists to align the 2 sets of images. Nevertheless, attempts have been made to coregister independently acquired PET and CT images in the thorax and abdomen $(6,7)$, and some success has been achieved by using reference markers and by operator intervention to guide the alignment (8). However, a definitive solution to this problem is to acquire both functional (PET) and anatomical (CT) images sequentially with a single scanner, without removing the patient from the bed.

This article describes the design and performance of a novel combined PET/CT scanner. Precisely co-registered anatomical and functional images can be acquired in a single scanning 
session, offering the potential for increased diagnostic accuracy. Patient positioning, attenuation correction, and scatter correction are based on the CT transmission images. The CT scan can be acquired postinjection without contamination from the injected activity, thus improving patient comfort and throughput. The combined PET/CT scanner has recently been installed in a hospital environment and is undergoing clinical evaluation. Three patient studies are presented to illustrate the scanner performance for applications in clinical oncology.

\section{MATERIALS AND METHODS}

\section{Design Concept}

The PET/CT scanner is based on the combination of a spiral CT scanner, a Somatom AR.SP (Siemens, Iselin, NJ), with the PET components from a rotating partial-ring tomograph, an ECAT ART scanner (Siemens). Both the PET and CT components are mounted on the same assembly, with the PET components on the reverse side of the rotating support of the CT scanner, as shown schematically in Figure 1A. The entire assembly is housed inside a single gantry, with the centers of the 2 tomographs offset axially by $60 \mathrm{~cm}$. The bed is installed at the front of the combined gantry and is used for both the PET and CT imaging. Bed travel allows dual-modality PET and CT images to be acquired for an axial extent of $100 \mathrm{~cm}$, sufficient to cover the range from chin to lower thigh in most patients.

The CT is a third generation helical scanner, a Somatom AR.SP (Fig. IB). Compared with single-slice CT, helical CT acquires multiple axial slices by a continuous motion of the patient bed. This results in shorter scan times and lower overall dose to the patient. Some relevant design parameters of the Somatom AR.SP are summarized in Table 1. The scanner has a metal ring M-CT 141 tube that produces $\mathrm{x}$-ray spectra of $110 \mathrm{kV}_{\mathrm{p}}$ or $130 \mathrm{kV}_{\mathrm{p}}$ with a $6.5 \mathrm{~mm} \mathrm{Al-equivalent} \mathrm{filter.}$ The tube is operated with a flying spot, and, thus, 1024 detectors can be read from 512 xenon gas-filled Quantillarc chambers. The x-ray tube, cooling system, detectors, and readout electronics are all mounted on the rotating support of the CT scanner. The packing density of these components precludes the possibility to mount the PET detectors on the same side of the rotating support as the CT. Instead, the PET components are mounted to the rear of the CT support ring, on a separate aluminum annulus attached to the CT support. The PET components include the detectors and electronics, coincidence processor and the optically coupled data transmitters. An asynchronous motor rotates the entire assembly of PET and CT components at $30 \mathrm{rpm}$.

The PET detectors are standard ECAT ART components. The ECAT ART is a partial-ring, rotating tomograph comprising dual arrays of BGO block detectors. Each array consists of 11 blocks (transaxially) by 3 blocks (axially), covering an arc of $83^{\circ}$. The detector arrays are not symmetrically opposed (Fig. 1C) but are offset by $15^{\circ}$ to increase the effective diameter of the transaxial field of view to $60 \mathrm{~cm}$ without requiring additional detector blocks. The detector blocks are $54 \mathrm{~mm} \times 54 \mathrm{~mm} \times 20 \mathrm{~mm}$ in size, cut into $8 \times 8$ crystals each of dimension $6.75 \mathrm{~mm} \times 6.75 \mathrm{~mm} \times 20 \mathrm{~mm}$. Thus, the axial field of view is $16.2 \mathrm{~cm}$ ( 24 partial rings of $6.75 \mathrm{~mm}$ ). Additional shielding of the PET components from out-of-field activity is provided by arcs of lead, $2.5 \mathrm{~cm}$ thick, mounted on both sides of the detector assembly and projecting $8.5 \mathrm{~cm}$ into the field of view beyond the front face of the detectors. Some basic design parameters of the ECAT ART components in the combined PET/CT tomograph are summarized in Table 2 .

The entire rotating assembly is housed within a single gantry 170 $\mathrm{cm}$ wide and $168 \mathrm{~cm}$ high. As shown in Figure 1A, the overall tunnel length is $110 \mathrm{~cm}$, with a $60 \mathrm{~cm}$ axial displacement between the centers of the CT and PET imaging fields of view. As a consequence of the

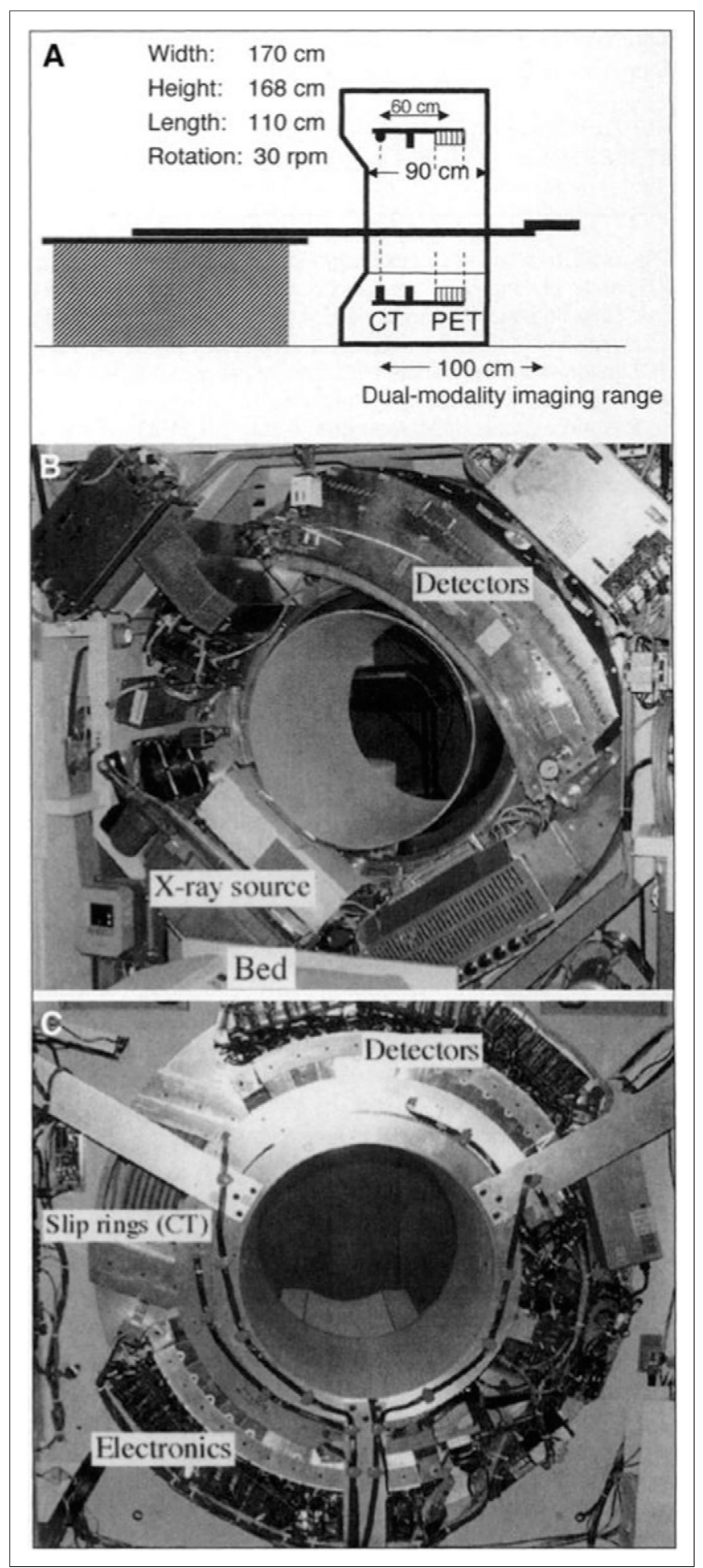

FIGURE 1. (A) Schematic of design concept of combined PET/CT scanner. PET components are mounted on aluminum disk attached to back of rotating CT scanner assembly. Centers of fields-of-view of PET and CT (vertical lines) are $60 \mathrm{~cm}$ apart. Combined PET/CT gantry is $110 \mathrm{~cm}$ deep, $170 \mathrm{~cm}$ high, and $168 \mathrm{~cm}$ wide. PET and CT data can be acquired over 100 $\mathrm{cm}$ axial range of patient. (B) Front view of Somatom AR.SP CT scanner (and front of combined PET/CT scanner) showing $x$-ray tube housing and $x$-ray detector assembly. Part of patient bed pallet also is seen. Note that space between $x$-ray tube and $x$-ray detectors is insufficient to accommodate 2 opposing ECAT ART detector arrays. (C) View from rear of PET/CT gantry showing PET detector arrays and electronics; ART detector electronics were rotated sideways $90^{\circ}$ to fit within CT gantry dimensions. Mechanical slip rings of CT also are labeled. Other modifications are described in text. 
TABLE 1

Design Characteristics of the Somatom AR.SP (CT) Components

\begin{tabular}{ll}
\hline \multicolumn{1}{c}{ Component } & \multicolumn{1}{c}{ Characteristics } \\
\hline Tube voltage & $110,130 \mathrm{kV}_{\mathrm{p}}$ \\
\hline Tube current & $63,83,105 \mathrm{~mA}$ \\
\hline Scan time per slice & $1.3,1.9 \mathrm{~s}$ \\
\hline Slice thickness & $1,2,3,5,10 \mathrm{~mm}$ \\
\hline Gantry aperture & $600 \mathrm{~mm}$ \\
\hline Focus to isocenter & $890 \mathrm{~mm}$ \\
\hline Fan beam opening & $52.2^{\circ}$ \\
\hline Transaxial field of view & $450 \mathrm{~mm}$ \\
\hline
\end{tabular}

extended length of the gantry, the tilt capability of the CT was disabled. A new, strengthened tunnel cover extending from the CT field of view to the rear gantry support was installed. The lasers for the PET imaging volume and the CT encoder are mounted on the outer surface of the cylindrical tunnel. The encoder operates optically, with a copper ring containing 2048 slots passing through the encoder slit. The position information of the $\mathrm{x}$-ray tube is obtained from the number of angular pulses generated by the slotted ring running through the optical encoder light switch as the assembly rotates. The zero position of the $\mathrm{x}$-ray tube is determined from an index slot on the encoder ring. The CT frequency controller was adjusted to take into account the slower acceleration of the gantry that was due to the additional load of the PET components. The positional information from the CT encoder is shared with the ART communication controller so that the lines of response (LORs) from the PET acquisition are correctly assigned in the sinogram.

Power supply and data transfer to and from the PET and CT components follow separate paths. The input voltage for the $\mathrm{x}$-ray tube is transformed from $110 \mathrm{~V}$ to $500 \mathrm{~V}$ outside the gantry and transmitted over mechanical slip rings. Power and serial communications to the ART components are transmitted over a different set of mechanical slip rings mounted with the ART detector arrays. High-speed digital data transfer from the ART is by optical transmission.

As with the standard ART scanner, the combined PET/CT scanner rotates continuously, eliminating the need for additional gantry cooling for the PET components. Fans start automatically if, for any reason, gantry rotation is halted. The CT scanner is cooled by 2 fans in the upper comers of the gantry that operate at all times. The x-ray tube is oil cooled.

TABLE 2

Design Characteristics of the ECAT ART (PET) Components

\begin{tabular}{ll}
\hline \multicolumn{1}{c}{ Component } & \multicolumn{1}{c}{ Characteristics } \\
\hline BGO block size & $54 \times 54 \times 20 \mathrm{~mm}$ \\
\hline Detector dimensions & $6.75 \times 6.75 \times 20.0 \mathrm{~mm}$ \\
\hline Crystal rings & 24 axial \\
\hline Axial field of view & $162 \mathrm{~mm}$ \\
\hline Detector ring diameter & $824 \mathrm{~mm}$ \\
\hline Image plane spacing & $3.375 \mathrm{~mm}$ \\
\hline Transaxial field of view & $600 \mathrm{~mm}$ \\
\hline
\end{tabular}

\section{Bed Support}

The Siemens CT bed and pallet, mounted to the front of the scanner as shown schematically in Figure 1A, is used to support the patient. Subjects up to $200 \mathrm{~kg}$ can be supported and positioned in the gantry to an accuracy of $\pm 0.5 \mathrm{~mm}$. To position the patient in both the CT and PET imaging fields, extended bed travel is required in addition to a support mechanism to prevent vertical deflection of the bed. The extended bed travel is required because of the $60 \mathrm{~cm}$ axial displacement between the CT and PET imaging fields of view. The use of an extended pallet allows an axial range of $100 \mathrm{~cm}$ (Fig. 1 A) to be scanned by both CT and PET, sufficient to cover most patients from chin to upper thigh. To ensure accurate alignment between the 2 imaging modalities, the patient bed should be supported throughout the length of the tunnel.

\section{Singles Transmission Sources}

To allow the combined scanner to be operated as a PET scanner only, additional transmission sources were incorporated into this prototype design. Dual, $550 \mathrm{MBq}{ }^{137} \mathrm{Cs}$ sources are mounted at opposite ends of the 2 PET detector arrays and transmission data are acquired in singles mode (9-11). The availability of singles transmission sources also enables a comparison to be made between CT-based attenuation correction factors and standard PET attenuation correction factors.

\section{PET/CT Image Processing}

The CT and PET components of the combined scanner are operated independently from separate consoles. The Somatom CT console, a SUN workstation, is used for CT image acquisition and reconstruction, and it also can be used for image display and evaluation, such as measurements of tumor size.

The $512 \times 512$ CT images are transferred over an Ethernet connection to the PET console, where combined PET/CT image processing is performed. The PET console comprises a $300-\mathrm{MHz}$ UltraSparc processor with 1 Gbyte RAM, a 2 Gbyte local disk, and a 9 Gbyte external disk. Before PET image reconstruction, the emission sinogram data are corrected for scatter and attenuation. Attenuation correction is based on a rescaling of the CT image (12) and scatter correction on a single scatter model (13). PET image reconstruction of the corrected 3-dimensional sinogram data is based on the Fourier rebinning algorithm FORE (14) followed by 2-dimensional iterative reconstruction using the ordered-subset EM approach (OSEM) (15). The specific implementation of the FORE + OSEM reconstruction procedure has been described elsewhere (16).

The reconstructed PET and CT images are viewed in the pixel resolution $(512 \times 512)$ of the $\mathrm{CT}$ image on the PET console. A tool has been developed to display transverse, coronal, and sagittal sections of the PET and CT image volumes, either adjacently with linked crosshairs or in fused mode with the PET images superimposed on the CT images. For fused image display, an interlaced pixel approach (17) is used with CT images in grayscale and the PET images superimposed in color (hot metal). The display scale of each image in both separate and fused mode can be adjusted independently. Zooming and regionof-interest capabilities are also provided.

\section{Operating Characteristics}

The performance of BGO-based PET detectors is affected by temperature fluctuations within the scanner environment (18). Temperature fluctuations inside the combined PET/CT gantry caused by heat dissipation from the operation of the $\mathrm{x}$-ray tube could be significantly greater than normal variations within the controlled gantry environment of current PET scanners. To investigate such effects, remote temperature sensors were placed at 4 locations within the combined gantry, 2 in opposite comers on the CT side and 2 in opposite 
comers on the PET side. Measurements were made of internal gantry temperature changes as a function of the operation of the CT scanner. Temperature readings were taken for all 4 sensors every $30 \mathrm{~min}$ for a period of $6 \mathrm{~d}$. The PET and CT detector assembly was rotated each day for $12 \mathrm{~h}$. For the first $4 \mathrm{~d}$, there was no operation of the x-ray source to determine the temperature stability of the gantry during rotation of the detectors. On day 4, a sequence of CT scans of a uniform cylinder was performed delivering about $4000 \mathrm{mAs}$ in total. On day 5, an extended series of both single and spiral CT scans was performed delivering a total of about 10,000 mAs.

Measurements of radiation dose delivered to the patient during a spiral CT scan were made using an MDH 9015 electrometer with a 10 $\mathrm{cm}$ pencil ionization chamber. Cylindrical phantoms of $15 \mathrm{~cm}$ and 30 $\mathrm{cm}$ diameter acrylic were used to simulate head (and extremities) and whole body. The multiple scan average dose (MSAD) at a depth of 1 $\mathrm{cm}$ was measured for typical CT scan parameters. Isodose curves for exposure levels around the tomograph were also obtained using an acrylic body phantom. To comply with radiation safety regulations and minimize exposure to personnel, the scanner room was modified to incorporate a lead glass window and lead shielding on the walls and access door.

\section{Performance Measurements}

The performance characteristics of the PET and CT components combined were anticipated to be the same as the performances of the ECAT ART and Somatom AR.SP, respectively. A summary of the performance of the ECAT ART based on the standard NEMA protocol (19) can be found elsewhere $(20,21)$. To verify that the ART components in the combined scanner have equivalent performance to a commercial ECAT ART, spatial resolution, energy resolution, scatter fraction, and counting rate performance were measured according to the NEMA standards.

The performance of the CT components was evaluated according to standard procedures for clinical CT scanners. These procedures include determining the attenuation values for air and water, and measurements of image pixel noise and spatial resolution. The attenuation value of water was measured using the test protocol of the Somatom AR.SP. A $20 \mathrm{~cm}$ diameter, water-filled cylinder was placed in the center of the field of view and images were acquired at $110 \mathrm{kV}_{\mathrm{p}}$ and $130 \mathrm{kV}_{\mathrm{p}}$. The mean pixel value and standard deviation were calculated for a circular region of interest covering $80 \%$ of the area of the water phantom. A similar measurement was performed with no phantom in the field of view to determine the attenuation value for air. The values for water and air are defined to be $0 \mathrm{HU}$ (Hounsfield units) and $-1000 \mathrm{HU}$, respectively, and should be independent of the x-ray tube voltage.

The water phantom then was repositioned in the center of the field of view and images again were acquired at $110 \mathrm{kV}_{\mathrm{p}}$ and 130 $\mathrm{kV}_{\mathrm{p}}$. Mean CT numbers were determined for five $4 \mathrm{~cm}$ diameter, circular regions of interest, positioned with 1 central region of interest and 4 others equally spaced peripherally. The absolute value of the difference between the average CT numbers of the central test area and the CT numbers of the 4 peripherally-placed regions of interest is then the homogeneity at $110 \mathrm{kV}_{\mathrm{p}}$ and $130 \mathrm{kV}_{\mathrm{p}}$. The spatial resolution is determined by scanning a phantom comprising an air-filled cylinder with a thin metal wire along its major axis, positioned parallel to the main scanner axis. CT resolution is expressed in line pairs per $\mathrm{cm}$.

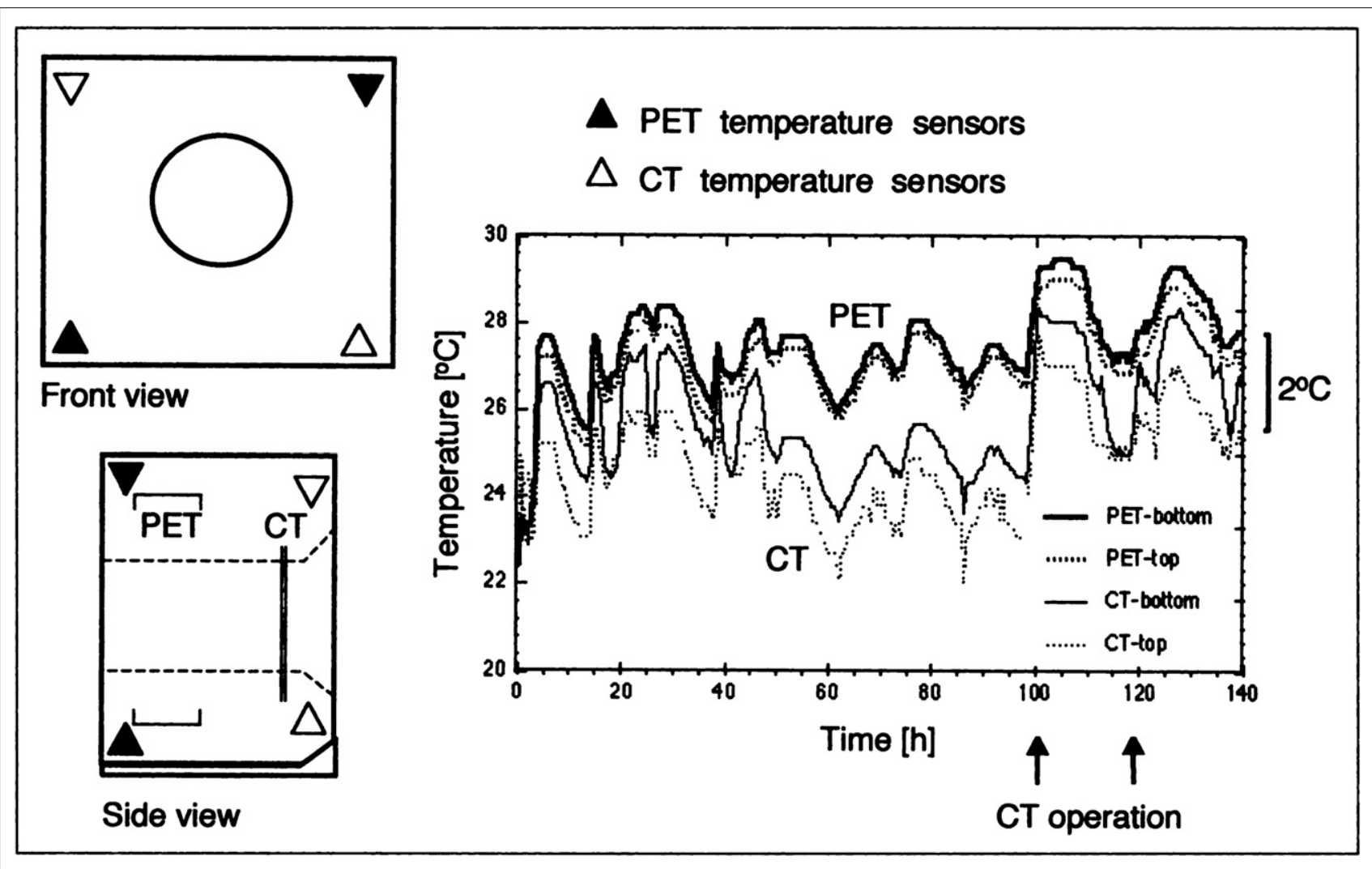

FIGURE 2. Gantry temperature $\left({ }^{\circ} \mathrm{C}\right)$ measured over $6 \mathrm{~d}$. Sensors were placed in top left and bottom right corners in front (CT) and rear (PET) of PET/ CT gantry. Between hours 0 and 144, gantry was rotated for 12 h every day. Hours 0-95: no x-ray exposure; hour 99: x-rays on ( 4,000 mAs); hour 120: x-rays on (10,000 mAs). 
For clinical, attenuation-corrected, whole-body imaging with FDG, it is important to acquire the transmission data postinjection to avoid having the patient occupying the scanner for the full 60 min uptake period required for FDG. To verify that the CT images are unaffected by the presence of positron-emitting activity in the patient, spiral CT images were acquired for two $20 \mathrm{~cm}$ diameter resin-filled cylinders, one containing no radioactivity and a second cylinder containing 130 $\mathrm{MBq}{ }^{68} \mathrm{Ge} /{ }^{68} \mathrm{Ga}$. The CT scans were acquired on the PET/CT scanner with a tube voltage of $130 \mathrm{kV}_{\mathrm{p}}, 160 \mathrm{mAs}$ and with a slice width of $10 \mathrm{~mm}$. Contiguous CT images were reconstructed, and $18 \mathrm{~cm}$ diameter regions of interest were centered over the cylinder in each slice. The region-of-interest mean and standard deviation for the cylinders with and without activity were compared to determine any effects that might be due to the activity in the cylinder and that would suggest emission contamination of the CT scan.

In a second study, three $5 \mathrm{~cm}$ diameter cylindrical inserts containing air and spongiosa and cortical bone-equivalent plastic were positioned in a $20 \mathrm{~cm}$ diameter, water-filled cylinder. Spiral CT images of the phantom were acquired in the Somatom AR.SP, without activity (preinjection) and with an activity concentration of $3.7 \mathrm{kBq} / \mathrm{mL}$ (postinjection) in the main compartment of the cylinder. The CT was acquired at $130 \mathrm{kV}_{\mathrm{p}}, 200 \mathrm{mAs}$, and with a $3 \mathrm{~mm}$ slice width and a pitch of 1.6. Contiguous $\mathrm{CT}$ images were reconstructed and circular, and $2.5 \mathrm{~cm}$ diameter regions of interest were placed over the inserts and the water cylinder. As for the previous study, the region-of-interest mean and SD were compared for each insert pre- and postinjection.

\section{Acquisition Protocols for Clinical Imaging}

For clinical imaging, a typical PET/CT acquisition protocol begins with a $260 \mathrm{MBq}$ injection of FDG and a 60 min uptake period. The patient is positioned in the scanner with the first transaxial section to be imaged aligned with the field of view of the CT. An initial scout scan (topogram) is performed to determine the axial range of the spiral scan. The maximum axial extent of a single spiral scan depends on the defined slice width and pitch. The total axial length to be scanned is subdivided into contiguous, $15 \mathrm{~cm}$ long segments. The spiral scan of each segment typically takes about $40 \mathrm{~s}$, and x-ray tube cooling sometimes may be required between segments. Patients are asked to hold their breath during the CT scan. For patients who cannot hold their breath for $40 \mathrm{~s}$, either shorter axial spirals are selected or the patients are instructed to breathe shallowly. The total time for the complete CT scan is 5-10 min. Once the spiral scans covering the full axial length are completed, the patient bed is moved to the start position of the multibed PET acquisition, and the PET scan is initiated. An emission scan time of $6-10$ min per bed position is selected depending on the number of bed positions, resulting in a total PET scan duration of 45-60 min. Typically, an axial overlap of $4 \mathrm{~cm}$ is used between contiguous bed positions.

\section{RESULTS}

\section{Operating Characteristics}

The results of the temperature measurements are summarized in Figure 2. Measurements were made over a period of $6 \mathrm{~d}$. The graph in Figure 2 shows that the temperature readings in the upper and lower parts of the PET scanner remained essentially constant during the first $96 \mathrm{~h}$ of the measurement period. Mean temperatures in the upper and lower parts were $26.8^{\circ} \mathrm{C} \pm 0.9^{\circ} \mathrm{C}$ and $27.1^{\circ} \mathrm{C}$ $\pm 1.0^{\circ} \mathrm{C}$, respectively. The measurements on the $\mathrm{CT}$ side of the scanner showed a slow steady decrease of about $1{ }^{\circ} \mathrm{C}$ over the same period; mean temperatures in the upper and lower parts were $24.2^{\circ} \mathrm{C}$ $\pm 0.9^{\circ} \mathrm{C}$ and $25.3^{\circ} \mathrm{C} \pm 1.0^{\circ} \mathrm{C}$, respectively. During the first $96 \mathrm{~h}$, temperature changes were due only to changes in ambient temperature and to changes related to the rotation of the gantry that occurred
TABLE 3

Multiscan Average Dose (MSAD) Measured for AR.SP at $1 \mathrm{~cm}$ Below Skin Surface for Spiral CT Scans

\begin{tabular}{lccc}
\hline Scan & Tube voltage $(\mathrm{kVp})$ & Flux $(\mathrm{mAs})$ & MSAD $(\mathrm{R})$ \\
\hline Head & 130 & 160 & 4.1 \\
Body & 110 & 200 & 2.1 \\
Body & 130 & 200 & 2.9 \\
Extremity & 110 & 160 & 2.8
\end{tabular}

Slice widths for head, body, and extremity scans were $10 \mathrm{~mm}$, $5 \mathrm{~mm}$, and $3 \mathrm{~mm}$ with pitches of 1,1.6, and 1.7, respectively.

for $12 \mathrm{~h}$ each day. At the end of both day 4 (96 h) and day 5 (122 h), the average temperature on die CT side increased to $26.0^{\circ} \mathrm{C} \pm 0.8^{\circ} \mathrm{C}$ and $27.0^{\circ} \mathrm{C} \pm 1.0^{\circ} \mathrm{C}$ in the upper and lower comers of the gantry, with similar temperature increases on the PET side, reaching $28.0^{\circ} \mathrm{C}$ $\pm 0.8^{\circ} \mathrm{C}$ and $28.4^{\circ} \mathrm{C} \pm 0.8^{\circ} \mathrm{C}$. Thus, the temperature increase on the PET side that was due to the operation of the $\mathrm{X}$-ray tube was $\sim 1^{\circ} \mathrm{C}$. A gradual decrease in temperature was noted after the $\mathrm{x}$-ray source was turned off.

$\mathrm{X}$-ray dose measurements are summarized in Table 3. The measurements were made with an acrylic head and body phantom and represent the single organ dose at a depth of $1 \mathrm{~cm}$ below the skin surface. The $\mathrm{x}$-ray tube was operated at scan conditions similar to those encountered in a clinical patient scan. Isodose curves are shown in Figure 3, representing constant exposure for a tube current product of $1000 \mathrm{mAs}$, equal to 5 scans at $100 \mathrm{~mA}$ with a $2 \mathrm{~s}$ scan time. The curves are skewed to the rear of the scanner and spread out to the front as a consequence of the asymmetric distribution of scattering media in the gantry.

\section{Performance Measurements}

The counting rate performance of the ECAT ART can be improved significantly by reducing the block integration time from $768 \mathrm{~ns}$ to $384 \mathrm{~ns}$, without any observable degradation in other

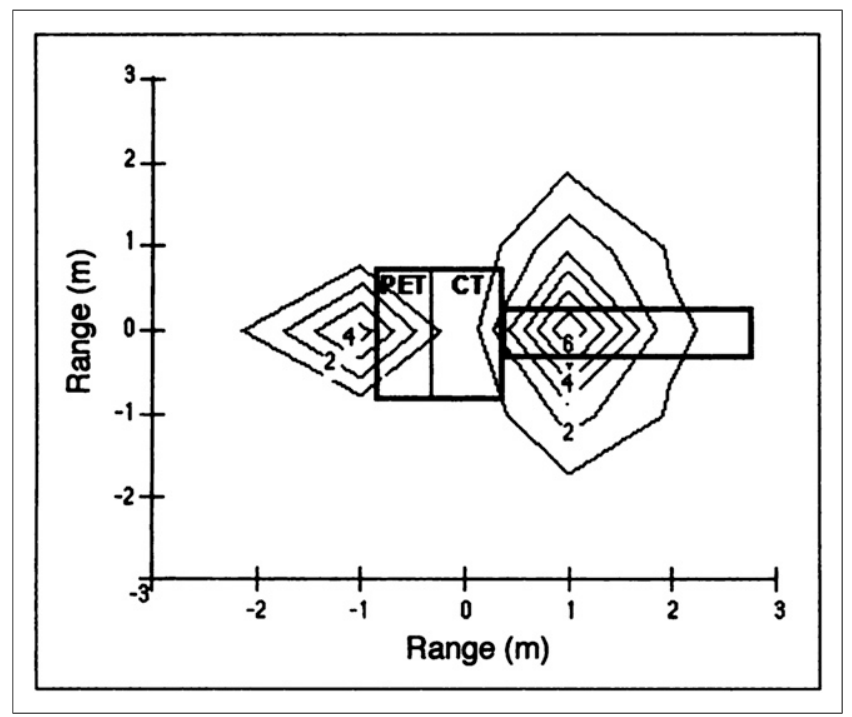

FIGURE 3. Isodose curves in $\mathrm{mR}$ for $1,000 \mathrm{mAs}$ scan measured in vicinity of PET/CT scanner. 
TABLE 4

Performance of Scanner PET Components

\begin{tabular}{ll}
\hline \multicolumn{1}{c}{ Component } & \multicolumn{1}{c}{ Performance } \\
\hline Transaxial spatial resolution & \\
at $r=0 \mathrm{~cm}$ & $6.2 \pm 0.3 \mathrm{~mm}$ \\
at $r=10 \mathrm{~cm}$ & $6.5 \pm 0.1 \mathrm{~mm}$ \\
Axial spatial resolution & \\
at $r=0 \mathrm{~cm}$ & $6.0 \mathrm{~mm}$ \\
Sensitivity & $8.4 \mathrm{cps} / \mathrm{Bq} / \mathrm{mL}$ \\
Scatter fraction & $0.36 \pm 0.02$ \\
Maximum NEC & $39.5 \mathrm{kcps}($ at $18 \mathrm{kBo} / \mathrm{mL})$ \\
\end{tabular}

Measurements based on NEMA protocol (19) with $384 \mathrm{~ns}$ block integration time and $12 \mathrm{~ns}$ coincidence time window.

performance parameters, such as spatial and energy resolution (21). Therefore, the ART components of the combined scanner are operated with a block integration time of $384 \mathrm{~ns}$. Standard performance measurements for the ART components in the combined scanner are summarized in Table 4 . The results were comparable with those for the standard ART scanner operated with a block integration time of $384 \mathrm{~ns}$ (21). The corresponding standard performance parameters for the Somatom AR.SP scanner are summarized in Table 5. Again, no degradation in performance was observed compared with a standard AR.SP.

The design of the prototype with the PET and CT components mounted on opposite sides of the aluminum support minimizes potential interference between the 2 imaging systems. The PET detectors are never exposed directly to the x-ray flux, and the operation of the CT has no residual effect on the photomultiplier tube gains. The PET components can be operated immediately after the acquisition of the $\mathrm{CT}$ scan without requiring any recovery time. However, the PET and CT components cannot acquire data simultaneously because of the high flux of scattered CT photons incident on the PET detectors that result in high levels of randoms and dead time caused by pulse pile-up. In view of the short time required for the $\mathrm{CT}$ scan, simultaneous operation of the PET and CT scanners is not considered necessary.

\section{Acquisition Protocols}

The importance of postinjection transmission scanning has been stressed previously. The aim is to avoid having the patient occupy

TABLE 5

\section{Performance of CT Components}

\begin{tabular}{ll}
\hline $\begin{array}{l}\text { Transaxial spatial } \\
\text { resolution }\end{array}$ & $0.45 \mathrm{~mm}$ (at $1.9 \mathrm{~s}$ scan time) \\
\hline CT value of air & $-1002 \pm 10 \mathrm{HU}$ \\
\hline CT value of water & $-2 \pm 4 \mathrm{HU}$ \\
\hline Cross-field uniformity & $<0.5 \mathrm{HU}(20 \mathrm{~cm}$ water cylinder $)$ \\
\hline Contrast scale & $(1.90 \pm 0.03) \cdot 10^{-4}$ \\
\hline Contrast resolution & $2.5 \mathrm{~mm} / 5 \mathrm{HU} / 1.9 \mathrm{~s}$ \\
\hline
\end{tabular}

Measurements based on standard service procedures for Somatom AR.SP. Siemens factory measurements of contrast scale and contrast resolution.

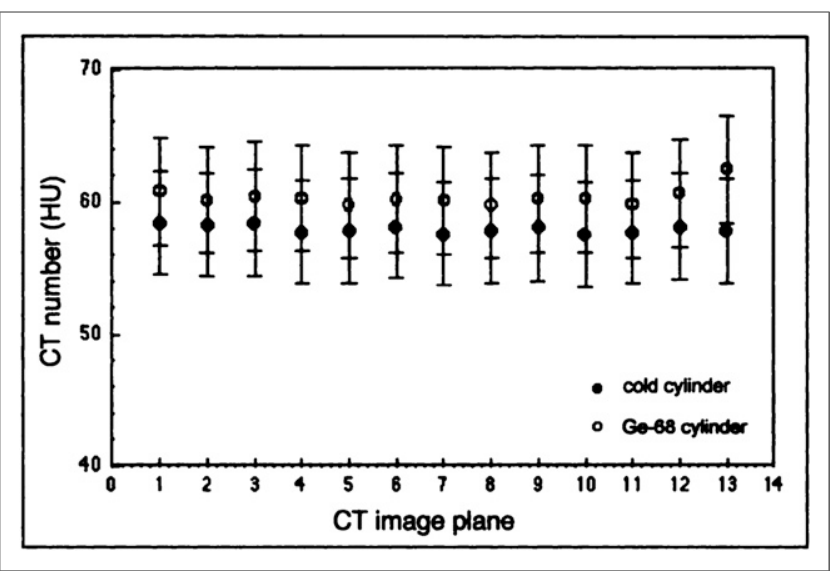

FIGURE 4. Mean region-of-interest values (HU) from set of transaxial $\mathrm{CT}$ images of cold (nonactive) and radioactive (130 MBq 68Ge) resinfilled uniform cylinder.

the scanner throughout the FDG uptake period, which would reduce patient throughput and increase the likelihood of patient movement between the transmission and emission scans. For the combined PET/CT scanner, postinjection transmission implies that the CT images must be unaffected by the presence of radioactivity in the patient. As described previously, 16 transverse CT sections were imaged along the axial length of a $20 \mathrm{~cm}$ diameter, uniform, resin-filled cylinder both without and with ${ }^{68} \mathrm{Ge}$ activity $(\sim 21 \mathrm{kBq} / \mathrm{mL})$ in the cylinder. The mean CT number within a circular region of interest placed on the image of the cylinder in each transaxial plane is plotted in Figure 4, both without and with activity in the cylinder. No contamination of the CT numbers by the additional activity in the cylinder is observed. The results of imaging the cylindrical phantom with the inserts are similar. The mean CT numbers for regions of interest placed over the inserts are summarized in Table 6 . As with the previous study, the CT numbers in the 3 inserts do not change in the presence of activity in the cylinder.

\section{Clinical Studies}

To illustrate the imaging of oncology patients on the combined PET/CT scanner, 3 representative case studies are presented. In each case, the patient was injected with $260 \mathrm{MBq}$ FDG, and an

\section{TABLE 6}

Attenuation Measurements Derived from CT Scans of 3 NEMA Phantom Inserts With and Without Emission Activity Contamination of $3.7 \mathrm{kBq} / \mathrm{ml}$ of $18 \mathrm{~F}$ Simulating Preinjection and Postinjection CT Scans

\begin{tabular}{lcc}
\hline \multicolumn{1}{c}{ Insert } & Preinjection & Postinjection \\
\hline Air & $-997.5 \pm 0.8$ & $-998.4 \pm 0.8$ \\
Spongiosa & $319.9 \pm 0.6$ & $319.0 \pm 1.0$ \\
Cortical & $1389 \pm 3$ & $1389 \pm 3$ \\
\hline
\end{tabular}

Mean region-of-interest values $(\mathrm{HU})$ were calculated from the central 37 transaxial image planes. Spiral CT scans were acquired at $110 \mathrm{kVp}, 160 \mathrm{mAs}, 3 \mathrm{~mm}$ slices, and a pitch of 1.6 . 
uptake period of $60 \mathrm{~min}$ was allowed before commencing the first spiral CT scan. For the patient studies presented here, spiral CT scans were acquired at $130 \mathrm{kV}_{\mathrm{p}}$ and $200 \mathrm{mAs}$ with a slice width of $10 \mathrm{~mm}$ and a pitch of 1.5 , unless stated otherwise. Contiguous CT images are reconstructed with a slice width of $3.4 \mathrm{~mm}$ corresponding to the default slice width for the PET scanner. The studies are presented in Figures 5-7. For each patient study, representative sections of the CT and PET volume images are displayed as (A) the CT image, (B) the corresponding PET image, and (C) the fused PET and CT image.

Case 1. This is a 78-y-old man with a history of T2, N2 squamous cell carcinoma of the right lung. A recent CT scan had demonstrated an increase in size of the right upper lobe mass, without lymphadenopathy. Metastatic work-up was negative preoperatively. The patient was referred for a PET/CT scan that was
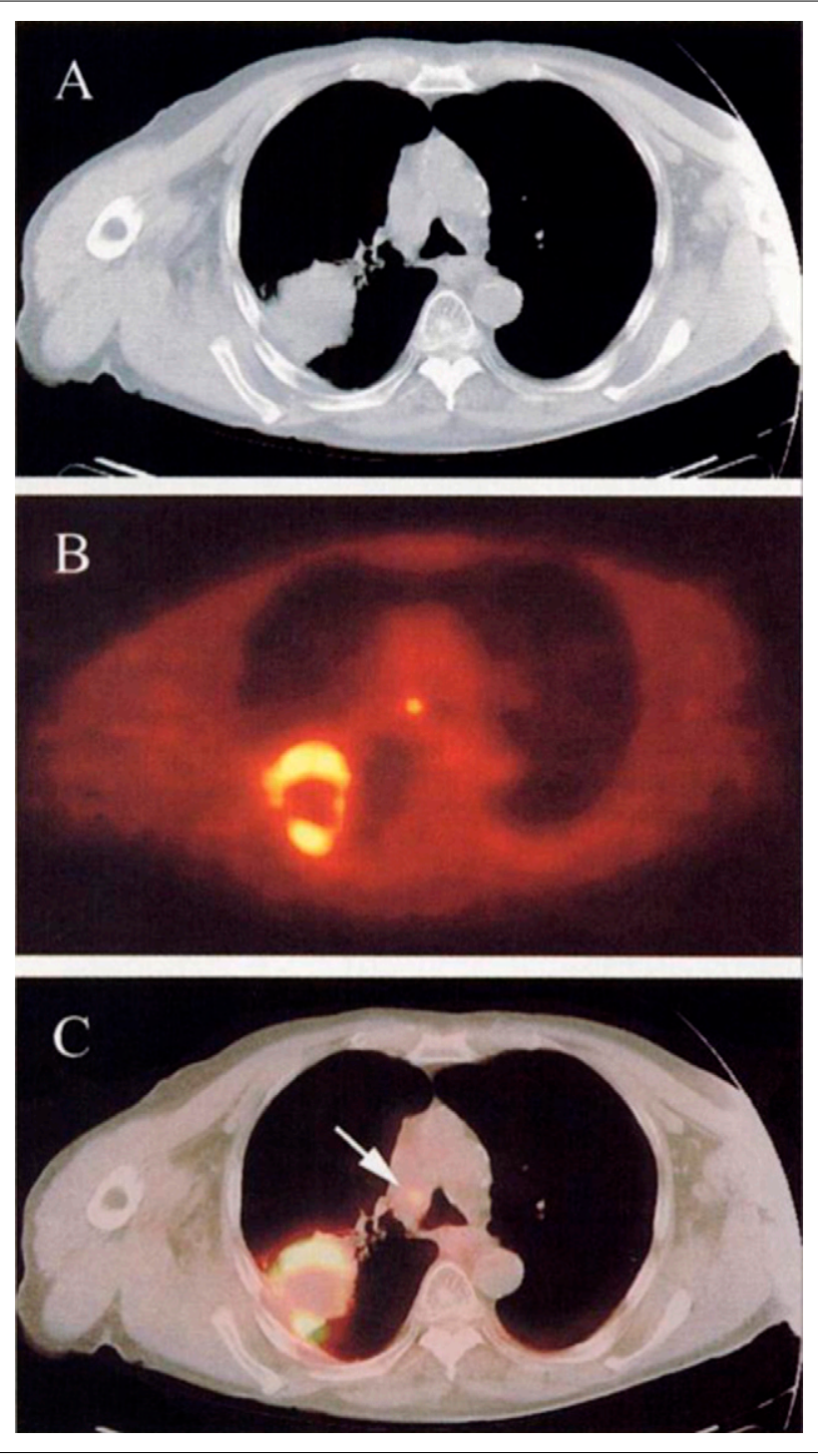

FIGURE 5. A 78-y-old man with squamous cell carcinoma of the lung. (A) Large isodense mass seen on CT appears on (B) PET scan as a hypermetabolic rim of increased FDG uptake, with necrotic center. (C) Fused image shows good alignment of 2 modalities. Lymph node in mediastinum (arrow) also demonstrated increased FDG uptake.
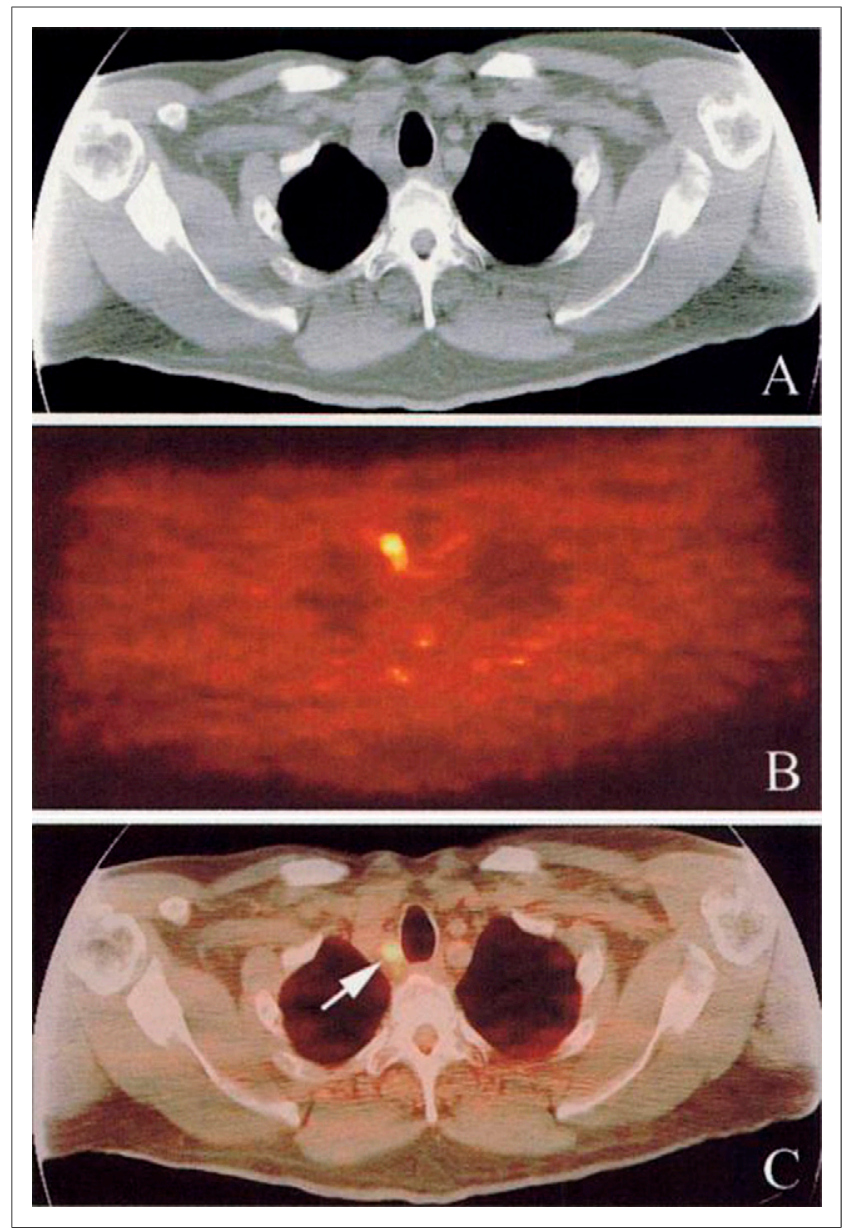

FIGURE 6. A 69-y-old man with diagnosed primary esophageal adenocarcinoma. (A) CT image. (B) PET image shows abnormal FDG uptake in the thorax. (C) Fused PET/CT image localizes uptake to specific lymph node (arrow).

performed over the thorax. The PET scan duration was 8 min per bed position. The images for this patient are presented in Figure 5. A large isodense mass in the upper lobe of the right lung appears on the fused image as a highly metabolic rim surrounding a necrotic center.

Case 2. A 69-y-old man presented with a 6-w period of dysphagia and odynophagia. A barium swallow examination was abnormal. He was diagnosed with a hiatal hernia and a distal esophageal defect just superior to the hernia. An esophagogastroduodenoscopy showed a malignant stricture at $36-40 \mathrm{~cm}$ and a duodenal polyp. The pathology report identified a signet-ring shaped adenocarcinoma. The patient was referred for a PET/CT scan, which was performed over the thorax. The PET scan duration was 10 min per bed position. The PET/CT images are shown in Figure 6. The fused image (Fig. 6C) clearly shows an area of increased FDG uptake in the lymph node.

Case 3. A 38-y-old woman with a history of unresectable pancreatic carcinoma was diagnosed in February 1998. A laparoscopy performed after placing a stent revealed liver metastases. The patient was referred for a PET/CT scan in August 1998. A wholebody scan was performed covering the thorax and abdomen. The PET scan duration was 10 min per bed position. The PET images (Fig. 7B) showed focal uptake in the abdomen that, from the fused 

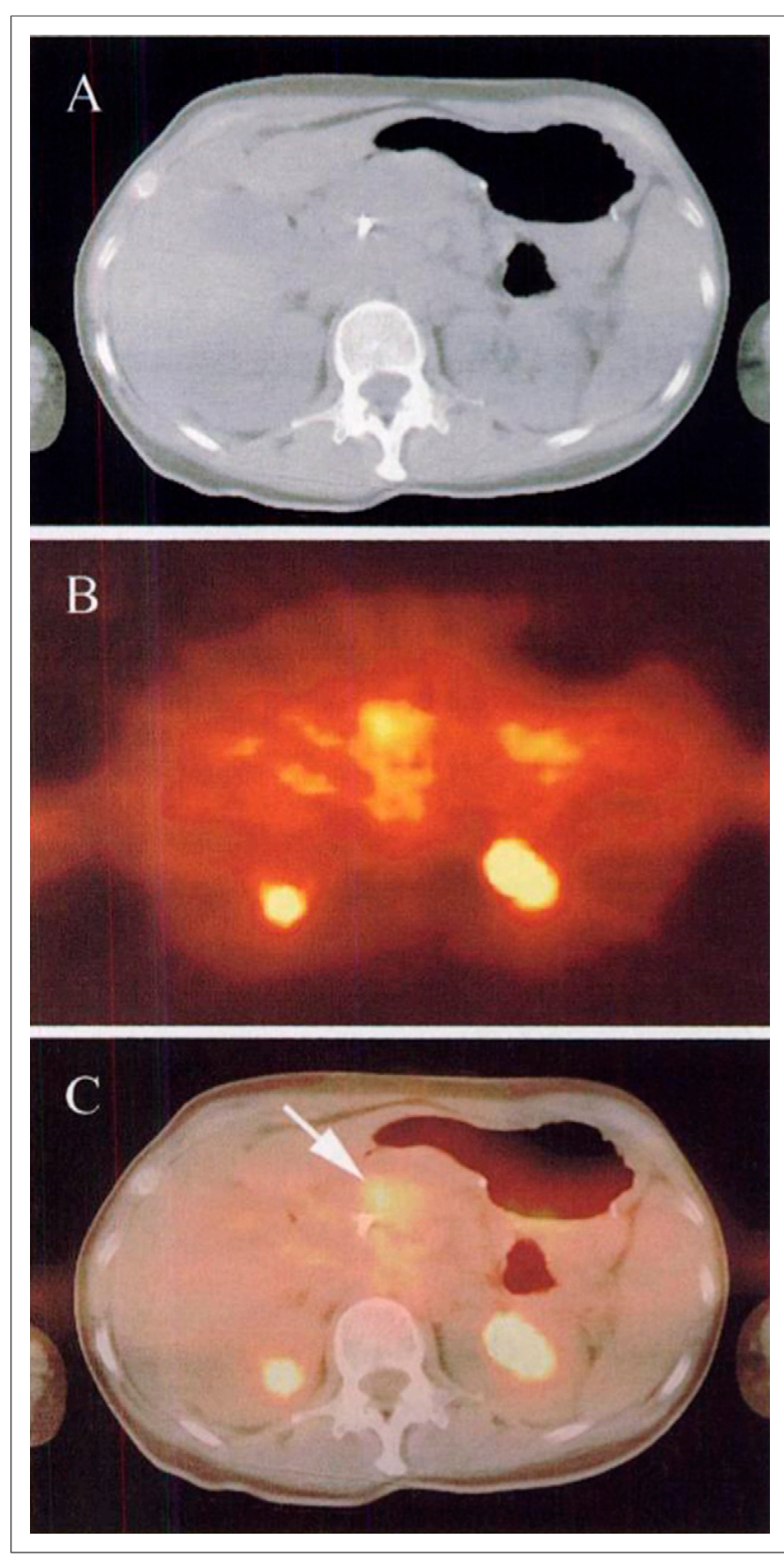

FIGURE 7. A 38-y-old woman with history of unresectable pancreatic cancer. Laparotomy revealed presence of liver metas-tases. (A) CT image. (B) Difficulty of accurately localizing FDG uptake can be seen from PET image. (C) Fused image enabled uptake to be localized to pancreas and not to transverse colon as had originally been thought.

image (Fig. 7C), was seen to be located in the pancreas; the uptake had an SUV of 5.3. Primary pancreatic cancer was confirmed for this patient during exploratory surgery.

\section{DISCUSSION}

The concept of dual-modality devices is not new. Lang et al. (22) developed a prototype CT/SPECT scanner using the same high-purity germanium detector array for both x-ray and singlephoton imaging. More recently, Hasegawa et al. (23) developed a CT/SPECT scanner by combining a commercial gamma camera with a CT scanner, and the device has been used for a small number of clinical studies. Interest in MR as the anatomical imaging modality led to the design of the first combined PET/MR scanner (24). Because of the significant difficulties of working within a high magnetic field, restricted volume environment, this approach has been limited to a single ring of PET detectors with an aperture suitable only for small animal imaging. Finally, a combined PET/SPECT detector is under development at CTI PET Systems (Knoxville, TN) based on a hybrid crystal comprising 2 scintillators, 1 for SPECT and 1 for PET. For SPECT imaging, conventional sodium iodide is used, whereas the recently discovered scintillator lutetium oxyorthosilicate (LSO) (25) is used for detecting $511 \mathrm{keV}$ photons.

The development of a combined PET and CT scanner is targeted primarily at oncology studies in the thorax and abdomen, although it also shows promise in cases of head and neck cancer. Wholebody oncology scans are performed to stage primary disease, evaluate nodal involvement, and identify metastatic spread to other organs. The lack of anatomical information in FDG PET scans frequently complicates interpretation, and considerable difficulty is encountered in accurately assigning functional abnormalities to specific anatomical structures. The potential for more tumorspecific tracers may eliminate even the low-resolution anatomical details discernible in FDG scans.

CT scans provide high-resolution, anatomical images that, in principle, can be aligned with the corresponding PET scan. Mathematical registration algorithms that are generally successful for the brain encounter more difficulty, however, in the rest of the body because of the movement of internal organs, differences in the profiles of the beds, and the lack of anatomical detail on either the PET emission or transmission scans. The use of external or internal reference markers does not resolve alignment problems resulting from variability in stomach and bowel contents and other uncontrolled internal physiological changes. The combined PET/ CT scanner addresses these issues by acquiring both anatomical and functional images in the same scanner, sequentially and closely spaced in time, and without moving the patient between die 2 scans.

One aspect of this design, as shown in Figure 1A, is that the centers of the CT and PET fields of view are separated axially by $60 \mathrm{~cm}$ and the overall tunnel length is $110 \mathrm{~cm}$. However, horizontal movement of a well-supported patient bed ensures a common scanning range of $100 \mathrm{~cm}$ without vertical deflection between the PET and CT images. No problems of claustrophobia or refusal to be scanned have been encountered among the 110 oncology patients scanned to date. The $60 \mathrm{~cm}$ axial separation is actually beneficial in minimizing potential interference between the PET and CT components, such as gantry temperature changes, as shown in Figure 2 and Tables 4 and 5. The PET and CT components, therefore, perform identically to the separate commercial systems the ECAT ART and the Somatom AR.SP. In a more integrated design where PET and CT components are mounted interleaved on the same side of the rotating support, however, issues of interference may be more significant and will have to be reevaluated.

For patient studies, the CT scan is acquired postinjection, immediately before the PET emission scan. The level of activity in the patient has no effect on the CT images (Fig. 4), and therefore no correction for contamination of the transmission data is required. Since the CT scan requires only about $6 \%-8 \%$ of the time for the PET emission scan, there is little to be gained by attempting to acquire the PET and CT scans simultaneously, particularly 
in view of possible artifacts in the PET data caused by CT photon pulse pile-up. Instead, a spiral CT is performed first with a continuous movement of the patient bed through the CT field of view (26). Once the spiral CT scan has been completed over the required axial range, the bed is moved into position within the PET field of view, and a PET acquisition for typically $10 \mathrm{~min}$ per bed position is commenced over the same axial range as the CT.

All PET images are corrected for attenuation using the CTbased correction algorithm (72). This algorithm is dependent on the choice of mean energy for the polychromatic (40 to $130 \mathrm{keV}$ ) CT x-ray beam. Generally, a mean energy of $70 \mathrm{keV}$ is used in deriving the appropriate scaling factors. Under standard operating conditions, the photon flux from a CT scanner is a factor of at least $10^{4}$ greater than that from conventional PET transmission sources. Thus, although the CT-based factors require scaling to $511 \mathrm{keV}$, compared with the standard PET transmission measurements with either rotating rod sources or point sources, the CT-based factors are essentially noiseless, even for obese, strongly attenuating patients.

The high-quality CT images also can be used to provide detailed anatomical information to the model-based scatter correction algorithm (13). In addition, the use of anatomical images as prior information to guide the PET reconstruction has been explored, and some results of this work using data from the combined PET/ CT scanner have been presented by Comtat et al. (27).

Several difficulties arise from the use of the CT images for attenuation and scatter correction. One obvious difficulty is that the spiral CT scan is acquired under breath-hold conditions, whereas the PET scan is acquired continuously over a 5 to $10 \mathrm{~min}$ period per bed position. The expansion of the chest during the CT scan may lead to misalignment of the PET and CT images in the thorax, resulting in incorrect PET attenuation correction factors, particularly for the anterior wall. One solution is to allow the patient to breathe during multiple rotations of the CT scan, although this may reduce the quality of the CT scan and introduce motion artifacts. For the PET attenuation correction factors, the high spatial resolution of the standard CT scan is not required, and hence some nonuniform blurring of the CT scan can be applied to provide a better match between the PET and CT images. Acquiring the CT scan with the patient in a state of expiration may be a third solution in the case of a cooperative patient.

Misalignment caused by respiration and cardiac motion is somewhat less of a problem in the abdomen. In this region of the body, combined anatomical and functional imaging is a powerful approach because of the intrinsic difficulty of interpreting PET images alone, as shown by the patient study in Figure 7. Abdominal imaging of patients with arms in the field of view often produces noticeable truncation and beam-hardening effects, particularly in obese subjects. The truncation is due to the acquisition of the CT scan with the arms of the patient in the field of view. The transaxial field of view of the CT scanner is $45 \mathrm{~cm}$, and CT scans of the abdominal or thoracic region generally are acquired with the arms up. This is standard procedure with the short CT imaging times, but the 40-50 min acquisition time for PET essentially precludes imaging with the arms up except for very cooperative patients. PET imaging with the arms down requires the full, $60 \mathrm{~cm}$ diameter transaxial field of view of the PET scanner. Therefore, the CT scan must be corrected for the truncated projections to avoid artifacts in the CT image and biases in the CT-based attenuation correction factors. These artifacts, and the effects of beam-hardening and intravenous or oral contrast on the CT-based attenuation correction algorithm, will be addressed in a separate publication.
The effectiveness of combined PET and CT imaging is illustrated by the selected clinical cases shown in Figures 5-7. The hypermetabolic rim surrounding the large lung tumor shown in Figure 5 appears on $\mathrm{CT}$ as an isodense mass. Accurate coregistration was achieved in this study despite respiratory motion, and the fused functional and anatomical images potentially provide important information to guide a biopsy of the lung mass to active regions of the tumor. In the case of esophageal cancer, the PET image in Figure 6 alone shows evidence for abnormal uptake of FDG that is difficult to localize to a particular lymph node. The fused image permits precise localization to a specific node that could result in a less invasive and more efficient surgical procedure. Figure 7 illustrates the importance of combined PET and CT imaging of the abdomen. The case is that of a woman with a surgically established primary pancreatic cancer. Using CT-based attenuation correction, the focal uptake in the head of the pancreas corresponded to an SUV of 5.3. The PET images alone of this region of the body are particularly difficult to interpret owing both to the absence of anatomical landmarks (other than kidneys) and the presence of nonspecific FDG uptake in stomach, bowel, and colon. These 2 examples illustrate the utility of fused PET/CT images for accurate interpretation of FDG uptake in the thorax and abdomen, and especially where caution should be exercised when associating abnormal FDG accumulation to a malignant process.

A thorough review of the clinical cases performed on the PET/ CT scanner and, in particular, an evaluation of the added value of the fused image have been published elsewhere (28).

The results from the prototype PET/CT scanner described here demonstrate the feasibility of acquiring co-registered PET and CT images in a patient during a single scanning session. The routine availability of both functional and anatomical images from a single scan offers significant advantages over acquiring the 2 modalities separately, particularly for oncology applications in the abdomen.

\section{CONCLUSION}

A prototype-combined PET/CT scanner was designed and built and is undergoing clinical evaluation. The scanner performs spiral whole-body CT scans and quantitative whole-body PET scans over a common axial range of $100 \mathrm{~cm}$ using low-noise, CT-based attenuation correction. The scans are acquired sequentially after a 60 min FDG uptake period. More than 110 patients, covering a wide range of different human cancers, have been scanned as of May 2000. The clinical results suggest an important role for combined PET and CT scanning in oncology. The acquisition of both functional and anatomical images, accurately aligned, in a single scanning session is convenient for the patient, simplifying the logistics and patient transfer. The combined PET/CT approach offers extensive possibilities for improving the diagnosis and staging of tumors, identification and localization of disseminated disease, improving radiotherapy treatment planning, and monitoring the effects of chemotherapy and radiation therapy.

\section{ACKNOWLEDGMENTS}

The authors thank Werner Ertel and Frank Schimmel from Siemens Erlangen (Germany) for their help and advice during the design period of the PET/CT. The authors are grateful to Marsha Dachille, James Ruszkiewicz, and Donna Milko from the PET Facility, University of Pittsburgh Medical Center, for their assistance during the installation and operation of the scanner; and Carolyn C. Meltzer, MD for her assistance in reading the scans. 
Doug Adams, Ken Baker, Thomas Bruckbauer, John Israel, Matthias Schmand, Keith Vaigneur, and Charles Watson are thanked for their extensive contributions during the design and construction phase of the combined scanner at CTI PET Systems. The authors gratefully acknowledge the contribution of Hugo Embert, a student from the Ecole Supérieure de Chimie Physique Electronique de Lyon, to the development of the fusion image tool. This work was supported by National Cancer Institute grants CA 65856 and CA74135. David W. Townsend is a consultant for CTI PET Systems, which manufactures the ECAT ART scanner. Tony Brun, Raymond Roddy, and John Young are employees of CTI Inc. Ronald Nutt is senior vice president and director of technology for CTI PET Systems. Larry Byars owns and directs Byars Consulting of Oak Ridge, TN.

\section{REFERENCES}

1. Smith TAD. FDG uptake, tumour characteristics and response to therapy: a review. Nucl Med Commun. 1998;19:97-105.

2. Strauss LG, Conti PS. The application of PET in clinical oncology. J Nucl Med. 1991;32:623-648.

3. Rigo P, Paulus P, Kaschten BJ, et al. Oncological applications of positron emission tomography with fluorine-18 fluorodeoxyglucose. EurJNucl Med. 1996;23: 1641-1674.

4. Eubank WB, Mankoff DA, Schmiedl UP, et al. Imaging of oncologic patients: benefit of combined CT and FDG PET in the diagnosis of malignancy. AJR 1998;171:1101-1110.

5. Woods RP, Cherry SR, Mazziotta JC. Rapid automated algorithm for aligning and reslicing PET images. J Comput Assist Tomogr. 1992;16:620-633.

6. Wahl RL, Quint LE, Cieslak RD, et al. Anatometabolic tumor imaging: fusion of FDG PET with CT or MRI to localize foci of increased activity. $J$ Nucl Med. 1993;34:1190

7. Tai YC, Lin KP, Hoh CK, Huang H, Hoffman EJ. Utilization of 3-d elastic transformation in the registration of chest $\mathrm{x}$-ray $\mathrm{CT}$ and whole body PET. IEEE Trans Nucl Sci. 1997;44:1606-1612.

8. Pietrzyk U, Herholz K, Heiss W-D. Three-dimensional alignment of functional and morphological tomograms. J Comput Assist Tomogr. 1990;14:51-59.

9. deKemp RA, Nahmias C. Attenuation correction in PET using single photon transmission measurement. Med Phys. 1994;21:771-778.

10. Watson CC, Jones WF, Brun T, Veigneur K. Design and performance of a single photon transmission measurement for the ECAT ART [CD-ROM]. IEEE Medical Imaging Conference Record. 1998.
11. Karp JS, Muehllehner G, Qu H, Yan XH. Singles transmission in volume-imaging PET with a ${ }^{137}$ Cs source. Phys Med Biol. 1995;40:929-944.

12. Kinahan PE, Townsend DW, Beyer T, Sashin D. Attenuation correction for a combined 3D PET/CT scanner. Med Phys. 1998;25:2046-2053.

13. Watson CC, Newport D, Casey ME. A single scatter simulation technique for scatter correction in 3D PET. In: Grangeat P, Amans J-L, eds. Computational Imaging and Vision. Dordrecht, The Netherlands: Kluwer Academic; 1996:255-268.

14. Defrise M, Kinahan PE, Townsend DW, et al. Exact and approximate rebinning algorithms for 3D PET data. IEEE Trans Med Imaging. 1997; 16:145-158.

15. Hudson H, Larkin R. Accelerated image reconstruction using ordered subsets of projection data. IEEE Trans Med Imaging. 1994;13:601-609.

16. Kinahan PE, Michel C, Defrise M, et al. Accelerated statistical reconstruction methods for PET and coincidence-SPECT whole-body oncology imaging [Abstract]. J Nucl Med. 1997;38:102P.

17. Rehm K, Strother SC, Anderson JR, et al. Display of merged multimodality brain images using interleaved pixels with independent color scales. J Nucl Med. 1994;35:1815-1821.

18. Reist HW, Stadelmann O, Kleeb W. Study on the stability of the calibration and normalization in PET and the influence of drifts on the accuracy of quantitation. Eur J Nucl Med. 1989;15:732-735.

19. Karp JS, Daube-Witherspoon ME, Hoffman EJ, et al. Performance standards in positron emission tomography. J Nucl Med. 1991; 12:2342-2350.

20. Bailey DL, Young H, Bloomfield PM, et al. ECAT ART - a continuously rotating PET camera: performance characteristics, initial clinical studies and installation considerations in a nuclear medicine department. Eur J Nucl Med. 1997;24:6-15.

21. Townsend DW, Beyer T, Join J, et al. The ECAT ART scanner for positron emission tomography: 1. Improvements in performance. Clin Pos Imag. 1998;2: 2-15.

22. Lang TF, Hasegawa BH, Liew SC, et al. Description of a prototype emission-transmission computed tomography imaging system. J Nucl Med. 1992;33:1881- 1887.

23. Hasegawa BH, Lang TF, Brown EL, et al. Object specific attenuation correction of SPECT with correlated dual-energy x-ray CT. IEEE Trans Nucl Sci. 1993; NS-40:1242-1252.

24. Shao Y, Cherry SR, Farahani K, et al. Simultaneous PET and MR imaging. Phy Med Biol. 1997;42:1965-1970.

25. Melcher CL, Schweitzer JS. Cerium-doped lutetium oxyorthosilicate: a fast, efficient new scintillator. IEEE Trans Nucl Sci. 1992;39:502-505.

26. Kalender WA, Seissler W, Klotz E, Vock P. Spiral volumetric CT with singlebreath-hold technique, continuous transport, and continuous scanner rotation. Radiology. 1990;176:181-183.

27. Comtat C, Kinahan PE, Fessler FA, et al. Reconstruction of 3D whole-body PET data using blurred anatomical labels. IEEE Medical Imaging Conference Record. 1999; CD-ROM.

28. Charron M, Beyer T, Bohnen N, et al. Whole-body FDG PET and CT imaging of malignancies using a combined PET/CT scanner [Abstract]. J Nucl Med. 1999;40: $256 \mathrm{P}$. 